\title{
Chalcones and Chalcone-mimetic Derivatives as Notch Inhibitors in a Model of T-cell Acute Lymphoblastic Leukemia
}

\author{
Deborah Quaglio, ${ }^{\dagger, O}$ Nadezda Zhdanovskaya, ${ }^{\ddagger}$, , Gloria Tobajas, ${ }^{\S}$ Viviana Cuartas, \\ Silvia Balducci, ${ }^{\dagger}$ Michael S. Christodoulou, ${ }^{\| \odot}$ Giancarlo Fabrizi, ${ }^{\dagger}$ Marta Gargantilla, ${ }^{\$}$
}

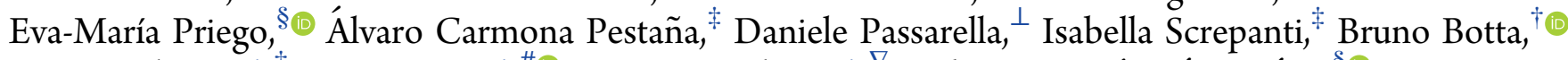
Rocco Palermo, ${ }^{* *}$ Mattia Mori, ${ }^{*},{ }^{\oplus}$ Francesca Ghirga, ${ }^{*, \nabla}$ and María-Jesús Pérez-Pérez ${ }^{\S}$

${ }^{\dagger}$ Department of Chemistry and Technology of Drugs, “Department of Excellence 2018-2022”, Sapienza Università di Roma, P.le Aldo Moro 5, 00185 Rome, Italy

${ }^{\ddagger}$ Department of Molecular Medicine, Sapienza University, 00161 Rome, Italy

${ }^{\S}$ Instituto de Química Médica, CSIC, Juan de la Cierva 3, 28006 Madrid, Spain

"DISFARM, Sezione di Chimica Generale e Organica “A. Marchesini”, Università degli Studi di Milano, Via Venezian 21, 20133 Milano, Italy

${ }^{\perp}$ Dipartimento di Chimica, Università degli Studi di Milano, Via Golgi, 19, 20133 Milano, Italy

\#Department of Biotechnology, Chemistry and Pharmacy, “Department of Excellence 2018-2022”, University of Siena, via Aldo Moro 2, 53100 Siena, Italy

${ }^{\nabla}$ Center for Life Nano Science@Sapienza, Istituto Italiano di Tecnologia, Viale Regina Elena 291, 00161 Rome, Italy

Supporting Information

ABSTRACT: Based on hit-likeness and chemical diversity, a number of chalcones and chalcone-mimetic compounds were selected as putative Notch inhibitors. The evaluation of the antiproliferative effect combined with the inhibition of Notch1 expression in KOPTK1 cell line identified compound 18, featuring a tetrahydronaphthalene-based scaffold, as a new promising Notch-blocking agent.

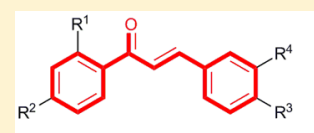

$1 R^{1}, R^{3}=-O H, R^{2}=-O_{3}, R^{4}=-C_{3}$ $5 \mathrm{R}^{1}, \mathrm{R}^{4}=-\mathrm{H}, \mathrm{R}^{2}=-\mathrm{CH}_{3}, \mathrm{R}^{4}=-\mathrm{OH}$ $8 R^{1}, R^{3}=O H, R^{2}=C_{3}, R^{4}=H$

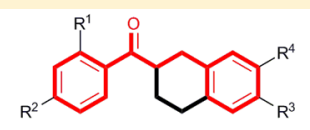

$9 R^{1}, R^{2}, R^{3}=-O_{C H}, R^{4}=-H$ $12 \mathrm{R}^{1}, \mathrm{R}^{3}=-\mathrm{OH}, \mathrm{R}^{2}=-\mathrm{OCH}_{3}, \mathrm{R}^{4}=-\mathrm{H}$ $18 R^{1}, R^{2}, R^{4}=-\mathrm{OCH}_{3}, R^{3}=-\mathrm{H}$

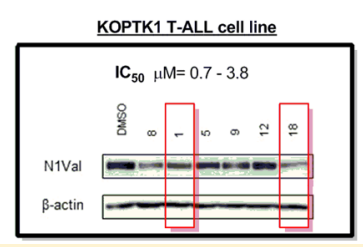

KEYWORDS: Chalcone, tetrahydronaphthalene scaffold, Notch inhibitors, T-ALL, cancer

$\mathrm{N}$ otch signaling is an evolutionarily conserved cell communication pathway regulating many biological processes including stem cells self-renewal, cell differentiation, proliferation, and survival in different tissues during embryonic and adult life. ${ }^{1-3}$ The mammalian Notch family includes four trans-membrane receptors (namely, Notch1-4), and five trans-membrane ligands (namely, Jagged-1 and -2, Delta-like$1,-3$, and -4). Notch signaling is a short-range intercellular communication system, wherein a membrane-tethered Notch ligand on the signal-sending cell interacts with a transmembrane Notch receptor on the juxtaposed signal-receiving cell. ${ }^{4}$ This activation induces a series of proteolytic events such as the $\mathrm{S} 2$ cleavage performed by the ADAM metalloproteases, and the S3 cleavage by the gamma secretase (GS) complex, which trigger the release of activated intracellular domain of Notch and its translocation to the nucleus. ${ }^{5}$ The subsequent interaction with the DNA binding protein CSL (CBF1/
Suppressor of Hairless/LAG ${ }^{-}$; also known as RBPJ) and Mastermind-like protein (MAML) promotes the transcription of Notch downstream target genes. ${ }^{5}$ Aberrant Notch activation due to either gene mutation or amplification or to posttranslational modifications has been linked to the onset of different solid and hematological cancers, including $\mathrm{T}$-cell acute lymphoblastic leukemia (T-ALL). Sixty percent of TALL cases are associated with activating NOTCH1 mutations and the majority of T-ALL patients shows increased expression and function of Notch3. ${ }^{6-8}$ Moreover, Notch signaling may contribute to chemotherapy resistance, thus suggesting Notch

Special Issue: Highlighting Medicinal Chemistry in Italy

Received: November 30, 2018

Accepted: February 26, 2019

Published: February 26, 2019 
Chart 1. Chemical Structures of Chalcone-8 Derivatives (1-7) and Chalcone Mimetics (9-18)

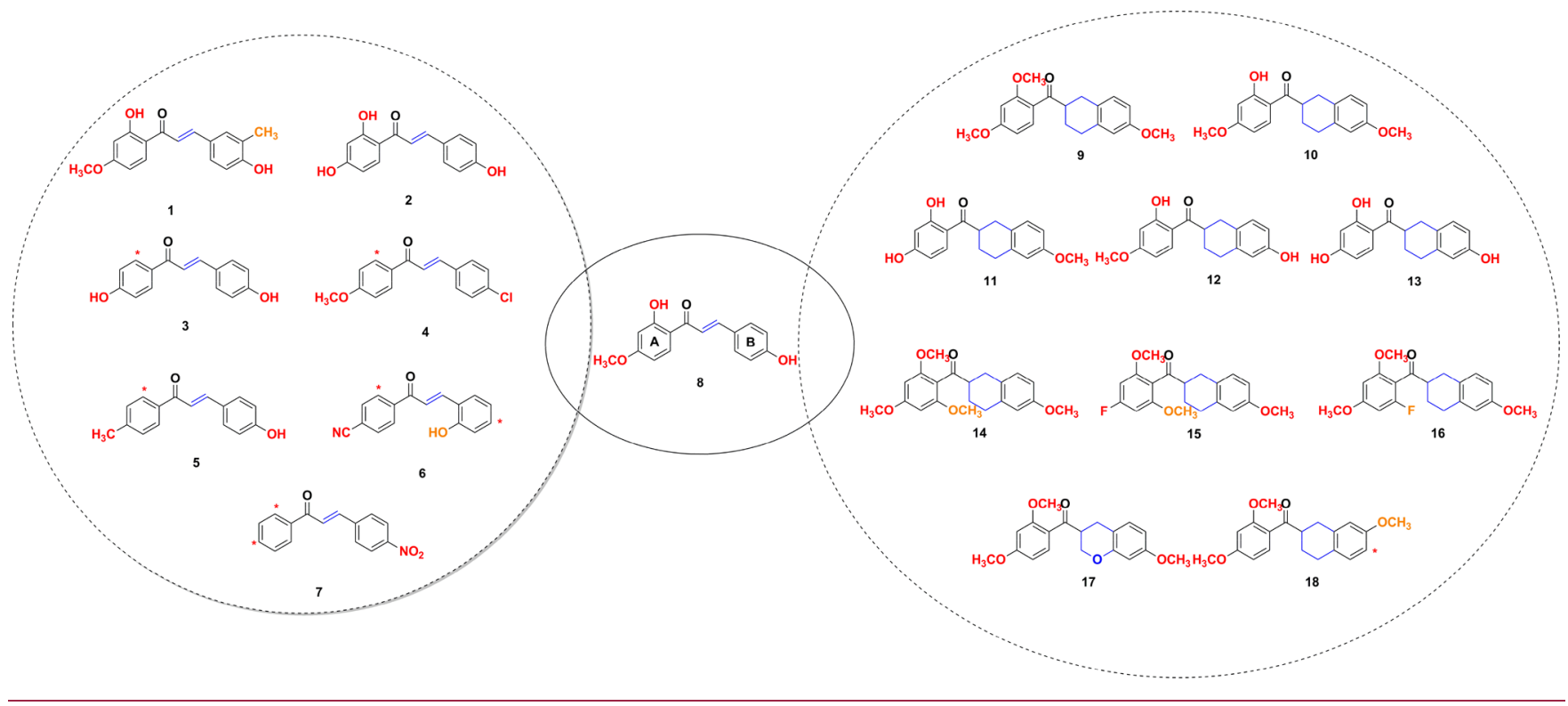

Scheme 1. Synthesis of the Chalcone mimetics $(9-18)^{a}$

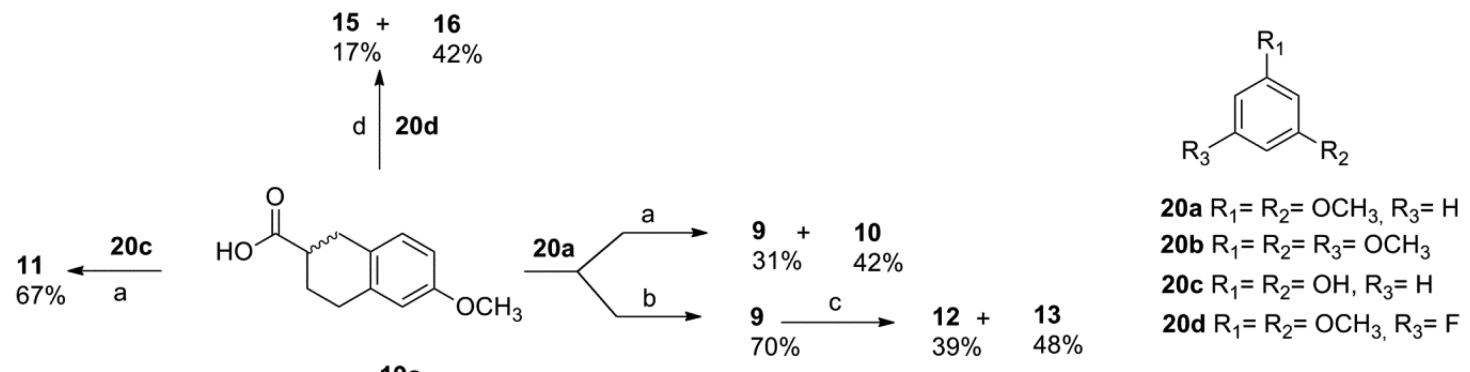

$19 a$

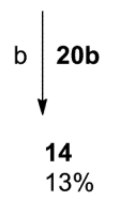

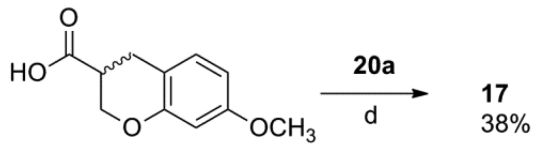

$19 b$

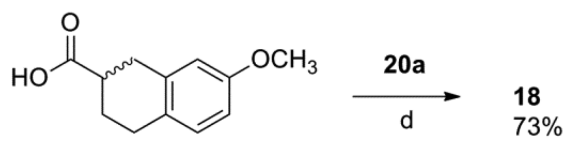

$19 c$

${ }^{a}$ Reagents and conditions: (a) (1) $\mathrm{SOCl}_{2}$, toluene, reflux, $3 \mathrm{~h}$; (2) $\mathrm{BF}_{3} \cdot \mathrm{OEt}_{2}, \mathrm{ClCH}_{2} \mathrm{CH}_{2} \mathrm{Cl}$ anh., $60{ }^{\circ} \mathrm{C}, 16 \mathrm{~h}$; (b) (1) SOCl , toluene, reflux, $3 \mathrm{~h}$; (2) TfOH, $\mathrm{ClCH}_{2} \mathrm{CH}_{2} \mathrm{Cl}$ anh., $60^{\circ} \mathrm{C}, 1 \mathrm{~h}$; (c) $\mathrm{BBr}_{3}$, anh. $\mathrm{CH}_{2} \mathrm{Cl}_{2}, 4{ }^{\circ} \mathrm{C}, 48 \mathrm{~h}$; (d) TFAA, TfOH, $\mathrm{ClCH}_{2} \mathrm{CH}_{2} \mathrm{Cl}$ anh, 15 min, rt.

inhibition as a strategy in anticancer targeted therapy. ${ }^{9,10}$ Notwithstanding, small molecules (e.g., GS inhibitors (GSI) such as MK-0752, RO4929097 and PF-03084014) and large molecules (e.g., Notch1-specific antibody OMP-52M51) currently in clinical development are not effective in all patients and often cause severe side-effects that limit their widespread therapeutic use and raise the need for novel Notchblocking agents. ${ }^{11,12}$

Recently, an in-house library of natural products and their derivatives was used as a source of potential inhibitors of the Notch signaling in T-ALL. ${ }^{13,14}$ Eight representative molecules of the library were selected through a cheminformatics approach and tested in vitro. The chalcone scaffold emerged as a promising tool to inhibit Notch signaling, and in particular, the synthesis of several chalcones combined with their biological evaluation highlighted the chalcone 8 as the most potent Notch blocking agent of the series, suggesting the synergistic activity of 2 ' - and 4-hydroxy groups. ${ }^{13,14}$ Herein, we designed and synthesized a number of chalcones and chalconemimetic compounds based on hit-likeness and chemical diversity, and we evaluated their antiproliferative activity in KOPTK1 cells. Small molecules able to reduce cell growth by more than $30 \%$ were further evaluated as inhibitors of Notch1 by means of Western blotting assay.

Results and Discussion. Synthesis of Chalcones and Chalcone-mimetic Derivatives. The general synthetic ap- 
proach to efficiently yield chalcone scaffold bearing substituents at different positions (1-7) was based on the Claisen-Schmidt condensation as previously reported (Chart 1). ${ }^{13,15}$ For the synthesis of the chalcone mimetics (9-18) (Chart 1$)$, the bicyclic acids $(19 a-c)$ were used to perform the Friedel-Crafts acylation of some arenes $(20 a-d)$ by using two different procedures (Scheme 1).

The ketone 9 was prepared by the reaction between the activated form of 1,2,3,4-tetrahydronaphthalene-2-carboxylic acid 19a with 1,3-dimethoxybenzene 20a in the presence of $\mathrm{BF}_{3} \cdot \mathrm{OEt}_{2}$. Since the catalyst induced the partial demethylation of the methoxy group vicinal to the ketone, the reaction afforded a mixture of 9 and 10 with $31 \%$ and $42 \%$ yields, respectively. Otherwise, performing the reaction with triflic acid, ${ }^{16}$ the ketone 9 was obtained in $70 \%$ yield. When resorcinol (20c) was used as the arene, compound 11 was obtained in $67 \%$ yield, so that the heating conditions favor the formation of the $C$-acylated product. ${ }^{17}$ Removal of the methoxy groups in 9 by using $\mathrm{BBr}_{3}{ }^{18}$ led to the formation of a mixture of 12 and 13 in $39 \%$ and $48 \%$ yields, respectively. The trimethoxy derivative $\mathbf{1 4}$ was obtained in low yield probably due to steric hindrance. However, the direct acylation reaction of the fluoroarene $20 \mathrm{~d}$ with the acid 19a by adapting previous reports using TFAA/TfOH, ${ }^{19,20}$ allowed the formation of the regioisomers $\mathbf{1 5}$ and 16, as shown in Scheme 1. Similarly, reaction of the 7-methoxychromane-3-carboxylic acid $19 \mathrm{~b}$ or 7-methoxy-1,2,3,4-tetrahydronaphthalene-2-carboxylic acid 19c with 20a in the presence of TFAA/TfOH afforded the aryl derivatives $\mathbf{1 7}$ and $\mathbf{1 8}$ in $38 \%$ and $73 \%$ yield, respectively. In all cases, compounds 9-18 were obtained as racemates.

Conformation of Chalcone 8 and Chalcone Mimetics. EChalcones can adopt $s$-cis or s-trans conformations. ${ }^{21}$ Nonsubstituted chalcones at the $\alpha$-position adopt, in general, a $s$-cis conformation, and this was confirmed for the chalcone 8 by 2D-NOESY experiments (Figure S1). The incorporation of a bicyclic ring in the structure of the chalcone mimetics (exemplified by compound $\mathbf{1 2}$ in Figure 1), where the double bond conjugated to the enone has been removed, is meant to restrict the conformational freedom of these analogues. The energy minimized conformers (see Computational Methods
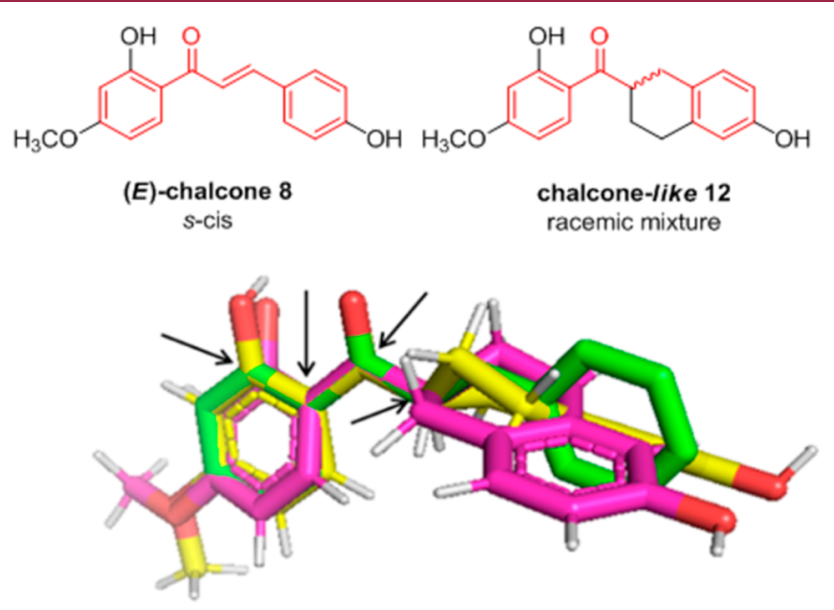

Figure 1. Superposition of the crystal structure of benzylidene acetophenone (green) with energy-minimized conformers of 12-S (magenta) and 12-R (yellow). Atom pairs used for the superimposition are indicated with black arrows.
S20) of the $12-R$ and $12-S$ enantiomers present in the racemic mixture were constructed and superimposed with the X-ray crystal structure of a model $s$-cis chalcone (that is, benzylidene acetophenone, CCDC 1185311). ${ }^{22}$ As shown in Figure 1, both enantiomers are able to locate the $\mathrm{OH}$-substituent on ring $\mathrm{B}$ close to the 4-position of ring B in the s-cis chalcone. Thus, the compounds were assayed as racemic mixtures.

Biological Results and Discussion. To evaluate the effectiveness of chalcones 1-7 and chalcone mimetics 9-18, we investigated their growth-inhibitory potency in KOPTK1 T-ALL cell line. These cells are sensitive to Notch inhibition by GSI and harbor activating Notch 1 gene mutations. ${ }^{7}$ As we demonstrated previously, in this cell line the reference compound 8 inhibited Notch 1 signaling and cell growth in the concentration range between 1 and $2.5 \mu \mathrm{M} .{ }^{13}$ Therefore, we compared the effects of compounds 1-7 and 9-18 in KOPTK1 cells on the endogenous levels of the activated domain of Notch1 (N1Val) by Western blotting and on cell growth by MTS assay, treating the cells with $2.5 \mu \mathrm{M}$ of each compound for $36 \mathrm{~h}$ and taking $30 \%$ decrease of cell viability as a threshold value for further screening (Figures 2a,b and S2). Notably, the comparison of the viability data and the N1Val levels after exposure to all chalcone derivatives confirmed a remarkably positive correlation between N1Val protein levels and the sensitivity of KOPTK1 cells to the bioactive compounds 1, 5, 9, 12, and 18 indicating that their antiproliferative effects were tightly associated with Notch signaling inhibition (Figure 2c). Further proving Notch inhibition, selected compounds decreased the N1Val protein expression and the endogenous mRNA levels of the Notch target gene DELTEX1 (DTX1). Interestingly, among them, only compounds 1 and $\mathbf{1 8}$ showed Notch1-inhibitory effects comparable to that of compound $\mathbf{8}$, whereas the Notch inhibition exerted by $\mathbf{5}, \mathbf{9}$, and $\mathbf{1 2}$ was weaker than that of compound $\mathbf{8}$ (Figures $2 \mathrm{~d}$ and S3). In addition, we determined the half-maximal growth-inhibitory concentration $\left(\mathrm{IC}_{50}\right)$ in a dose-response proliferation assay with increasing concentrations (namely, $0,0.5,1,2.5,5,10,20$, and $50 \mu \mathrm{M}$ ) of selected compounds 1, 5, 9, 12, and 18. These molecules inhibited leukemic cell growth to a similar extent each other, with $\mathrm{IC}_{50}$ values comparable to that of the reference compound 8 and ranging from 0.7 to $3.8 \mu \mathrm{M}$ (Figure 2e). Notably, statistical analysis of single-point comparative treatment showed no significant differences in the inhibition of cell growth between compounds $8,1,9$, and 18 , which provided a comparable effect at $2.5 \mu \mathrm{M}$ concentration. The slightly higher $\mathrm{IC}_{50}$ values of $\mathbf{5}$ and $\mathbf{1 2}$ are in line with their weaker effect on Notch1 inhibition (Figures 2a,d and S3). Determination of the $\mathrm{IC}_{50}$ value for compound $\mathbf{1}$ was not possible due to the irregular shape of its growth inhibition curve (Figure S4). Moreover, consistent with the effect induced by chalcone 8 in G1-phase cell cycle arrest and apoptosis, ${ }^{13}$ we found that growth inhibition by $\mathbf{1}$ was accompanied by both increased levels of the antiproliferative factor p27kip and of the apoptotic marker cleaved poly ADP-ribose polymerase (CL PARP). On the contrary, the most promising chalcone mimetics $\mathbf{9}$ and $\mathbf{1 8}$ failed to upregulate p27kip and promoted the highest levels of PARP cleavage among tested compounds (Figure 2f), whereas the weakest Notch inhibitors 5 and $\mathbf{1 2}$ induced p27kip without affecting PARP processing (Figure 2f). Finally, chalcone mimetics 9 and 18 affected cell proliferation also in GSIresistant JURKAT cell line $\mathrm{e}^{23}$ and in immortalized nontumorigenic human keratinocyte $\mathrm{HaCat}$ cells in which Notch 


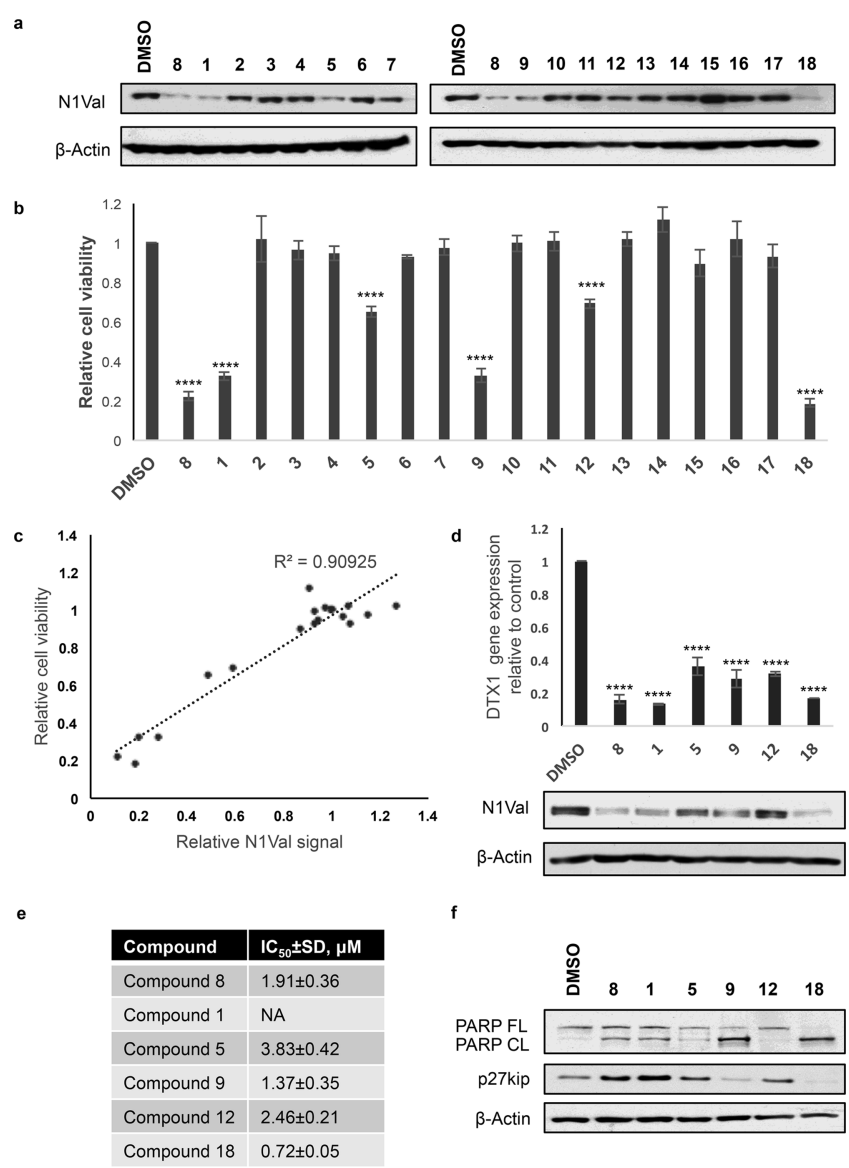

Figure 2. Antiproliferative and molecular effects of chalcones and chalcone-like derivatives in KOPTK1 T-ALL cells. (a) Protein expression levels of activated domain of Notch1 (N1Val) in KOPTK1 cells treated for $36 \mathrm{~h}$ with $2.5 \mu \mathrm{M}$ of compounds 1-18 or vehicle alone (DMSO). $\beta$-Actin is used as loading control. (b) Histogram shows the mean of relative cell viability of KOPTK1 cells treated with $2.5 \mu \mathrm{M}$ of compounds $\mathbf{1 - 1 8}$ or DMSO for $36 \mathrm{~h}$ measured with MTS assay \pm SEM obtained from two independent experiments performed in triplicate. $* * * * P<0.0001$, comparing with DMSO. (c) Representative graph showing the results of Pearson's correlation test between the average values of relative N1Val expression obtained with optical densitometry and cell viability in KOPTK1 treated with compounds $\mathbf{1 - 1 8}$ vs DMSO. $R=0.9535, R^{2}=$ $0.90925, p<0.0001$. (d) N1Val and $\beta$-actin protein expression levels (lower panel) and DELTEX1 (DTX1) relative gene expression levels (upper panel) in KOPTK1 cells treated with $2.5 \mu \mathrm{M}$ of compounds 8 , $1,5,9,12$, and 18 or vehicle alone (DMSO) for $36 \mathrm{~h}$. Data represent mean values for three independent experiments performed in triplicate \pm SEM. (e) IC $_{50}$ values of compounds $8,1,5,9,12$, and 18 determined in KOPTK 1 after $36 \mathrm{~h}$ of incubation. Data represent mean values for three independent experiments performed in triplicate \pm SD. (f) Protein expression of p27kip and of the nonprocessed PARP (FL PARP) and its cleaved form (CL PARP). $\beta$ Actin is used as loading control.

signaling acts as an onco-suppressor. ${ }^{24}$ These latter observations suggest different mechanisms of action and/or off-target effects by chalcones and chalcone mimetics (Figure S5).

$S A R$ of Chalcones and Chalcone-mimetic Derivatives as Notch Inhibitors. Structure-activity relationships (SAR) of a previous series of chalcone derivatives revealed how the $2^{\prime}$ - and 4-hydroxy groups are essential for both cell proliferation and Notch inhibition. ${ }^{13}$ The biological results obtained with the chalcones described in this work confirmed the previous SAR.
Accordingly, the effectiveness of the chalcones $\mathbf{1}$ and $\mathbf{5}$ on growth inhibition of KOPTK1 T-ALL cell line highlighted the pivotal role of $4-\mathrm{OH}$ substitution to ring $\mathrm{B}$ (Figure $2 \mathrm{~b}$ ). However, the Western blotting assay on N1Val expression using chalcone $\mathbf{8}$ as the reference compound showed a comparable inhibitory effect just for $\mathbf{1}$ (Figure 2a). These results reinforced the previous evidence also for the ring A showing how the lack of 4-OH substitution led to a weaker inhibitory activity toward activated Notch1. The introduction of $3-\mathrm{CH}_{3}$ group at ring $\mathrm{B}$ did not influence the inhibition of endogenous Notch signaling activity and cell growth.

Chalcone mimetics $9, \mathbf{1 2}$, and $\mathbf{1 8}$ proved to decrease the cell viability of KOPTK1 T-ALL cells with $\mathrm{IC}_{50}$ values in the lowup to submicromolar range (Figure $2 \mathrm{e}$ ). While compound 12, with a substitution pattern at rings $A$ and $B$ similar to that of the reference chalcone 8 , showed some Notch-inhibitory effects, the best inhibition of Notch1 intracellular domain in this series was obtained by compound $\mathbf{1 8}$ (Figure $2 \mathrm{~d}$ ). Thus, it is interesting to note that compounds incorporating a tetrahydronaphthalene scaffold are also able to exert inhibition of the Notch pathway, although with some differences compared to chalcones.

Conclusion. Based on previous evidence and molecular modeling studies, several chalcones 1-7 and chalcone mimetics 9-18 were selected, synthesized, and tested toward antiproliferative activity and Notch inhibition in KOPTK1 TALL cells. Compounds $\mathbf{1}$ and $\mathbf{1 8}$ exhibited cell growth reduction combined with inhibition of Notch1 intracellular domain, mostly comparable to the reference compound 8. SAR analysis of the chalcone series confirmed the key role played by the $2^{\prime}$ - and $4-\mathrm{OH}$ groups on both antiproliferative and Notch1 inhibitory activity. However, the introduction of bicyclic ring in the structure of the chalcone mimetics promotes Notch inhibition through different biological responses, thus suggesting that more in-depth investigations are needed to further develop tetrahydronaphthalene derivatives as Notch blocking agents.

\section{ASSOCIATED CONTENT}

\section{S Supporting Information}

The Supporting Information is available free of charge on the ACS Publications website at DOI: 10.1021/acsmedchemlett.8b00608.

Experimental details for synthetic procedures, associated chemical data for compounds 1-18, computational methods, and biological assays (PDF)

\section{AUTHOR INFORMATION}

\section{Corresponding Authors}

*(M.M.) Phone: +390577234256 E-mail: m.mattia79@gmail. com.

*(F.G.) Phone: +390649693318. E-mail: francesca.ghirga@iit. it.

*(R.P.) Phone: +390649255142. E-mail: rocco.palermo@ uniroma1.it.

\section{ORCID}

Michael S. Christodoulou: 0000-0002-5098-3143

Eva-María Priego: 0000-0001-9470-4508

Bruno Botta: 0000-0001-8707-4333

Mattia Mori: 0000-0003-2398-1254

María-Jesús Pérez-Pérez: 0000-0003-1336-7760 


\section{Present Addresses}

(V.C.) Grupo de Investigación de Compuestos Heterocíclicos, Departamento de Quimica, Universidad del Valle, A.A. 25360 Cali, Colombia. E-mail: viviana.cuartas@correounivalle. edu.co.

(N.Z.) Department of Physics, University of Rome "Sapienza", Rome, Italy.

\section{Author Contributions}

OThese authors contributed equally. The manuscript was written through contributions of all authors. All authors have given approval to the final version of the manuscript.

\section{Notes}

The authors declare no competing financial interest.

\section{ACKNOWLEDGMENTS}

The authors acknowledge networking contribution by the COST Action CM1407 "Challenging organic syntheses inspired by nature - from natural products chemistry to drug discovery". V.C. thanks the Universidad del Valle (CIAM2017) and the Science, Technology and Innovation FundGeneral Royalties System (FCTeI-SGR) under contract BPIN 2013000100007 for a predoctoral fellowship. This work has been supported by Italian Ministry of Education, University and Research - Dipartimenti di Eccellenza - L. 232/2016. This work has been partially supported by a grant from MINECO/ FEDER SAF2015-64629-C2-1-R (to M-J.P.-P and E-M.P), MIUR PNR 2015-2020 (ARS01_00432) (to I.S.), and Sapienza University 2016 Project number RG116154E2C7A6FB (to I.S.).

\section{ABBREVIATIONS}

ADAM, a disintegrin and metalloproteinase; GS, gamma secretase; GSI, gamma secretases inhibitor; CSL, CBF-1/SuH/ Lag-1; MAML, mastermind-like; T-ALL, T-cell acute lymphoblastic leukemia

\section{REFERENCES}

(1) Liu, J.; Sato, C.; Cerletti, M.; Wagers, A. Notch signaling in the regulation of stem cell self-renewal and differentiation. Curr. Top. Dev. Biol. 2010, 92, 367-409.

(2) Siebel, C.; Lendahl, U. Physiol. Rev. 2017, 97, 1235-1294.

(3) Koch, U.; Lehal, R.; Radtke, F. Stem cells living with a Notch. Development 2013, 140 (4), 689-704.

(4) Kopan, R.; Ilagan, M. X. The canonical Notch signaling pathway: unfolding the activation mechanism. Cell 2009, 137 (2), 216-33.

(5) Palermo, R.; Checquolo, S.; Bellavia, D.; Talora, C.; Screpanti, I. The molecular basis of notch signaling regulation: a complex simplicity. Curr. Mol. Med. 2014, 14 (1), 34-44.

(6) Bellavia, D.; Palermo, R.; Felli, M. P.; Screpanti, I.; Checquolo, S. Notch signaling as a therapeutic target for Acute Lymphoblastic Leukemia. Expert Opin. Ther. Targets 2018, 22 (4), 331-342.

(7) Weng, A. P.; Ferrando, A. A.; Lee, W.; Morris, J. P., 4th; Silverman, L. B.; Sanchez-Irizarry, C.; Blacklow, S. C.; Look, A. T.; Aster, J. C. Activating mutations of NOTCH1 in human T cell acute lymphoblastic leukemia. Science 2004, 306 (5694), 269-271.

(8) Bellavia, D.; Campese, A. F.; Checquolo, S.; Balestri, A.; Biondi, A.; Cazzaniga, G.; Lendahl, U.; Fehling, H. J.; Hayday, A. C.; Frati, L.; von Boehmer, H.; Gulino, A.; Screpanti, I. Combined expression of pTalpha and Notch3 in T cell leukemia identifies the requirement of preTCR for leukemogenesis. Proc. Natl. Acad. Sci. U. S. A. 2002, 99 (6), 3788-3793.

(9) Aster, J. C.; Pear, W. S.; Blacklow, S. C. The Varied Roles of Notch in Cancer. Annu. Rev. Pathol.: Mech. Dis. 2017, 12, 245-275.

(10) Palermo, R.; Ghirga, F.; Piccioni, M. G.; Bernardi, F.; Zhdanovskaya, N.; Infante, P.; Mori, M. Natural products inspired modulators of cancer stem cells-specific signaling pathways Notch and Hedgehog. Curr. Pharm. Des. 2019, 25, 1.

(11) Andersson, E. R.; Lendahl, U. Therapeutic modulation of Notch signalling-are we there yet? Nat. Rev. Drug Discovery 2014, 13 (5), 357-78.

(12) Takebe, N.; Nguyen, D.; Yang, S. X. Targeting notch signaling pathway in cancer: clinical development advances and challenges. Pharmacol. Ther. 2014, 141 (2), 140-9.

(13) Mori, M.; Tottone, L.; Quaglio, D.; Zhdanovskaya, N.; Ingallina, C.; Fusto, M.; Ghirga, F.; Peruzzi, G.; Crestoni, M. E.; Simeoni, F.; Giulimondi, F.; Talora, C.; Botta, B.; Screpanti, I.; Palermo, R. Identification of a novel chalcone derivative that inhibits Notch signaling in T-cell acute lymphoblastic leukemia. Sci. Rep. 2017, 7 (1), 2213.

(14) Botta, B.; Screpanti, I.; Tottone, L.; Zhdanovskaya, N.; Ingallina, C.; Giulimondi, F.; Quaglio, D.; Palermo, R.; Mori, M.; Ghirga, F. Inibitori di Notch per uso nel trattamento della leucemia linfoblastica acuta a cellule T. Domanda internazionale nr. PCT/ IB2017/058204 2017. Deposito 20-12-2017.

(15) Schiano Moriello, A.; Luongo, L.; Guida, F.; Christodoulou, M. S.; Perdicchia, D.; Maione, S.; Passarella, D.; Marzo, V. D.; De Petrocellis, L. Chalcone Derivatives Activate and Desensitize the Transient Receptor Potential Ankyrin 1 Cation Channel, Subfamily A, Member 1 TRPA1 Ion Channel: Structure-Activity Relationships in vitro and Anti-Nociceptive and Anti-inflammatory Activity in vivo. CNS Neurol. Disord.: Drug Targets 2016, 15, 987-994.

(16) Bueno, O.; Tobajas, G.; Quesada, E.; Estévez-Gallego, J.; Noppen, S.; Camarasa, M.-J.; Díaz, J.-F.; Liekens, S.; Priego, E.-M.; Pérez-Pérez, M.-J. Conformational mimetics of the $\alpha$-methyl chalcone TUB091 binding tubulin: Design, synthesis and antiproliferative activity. Eur. J. Med. Chem. 2018, 148, 337-348.

(17) Adogla, E. A.; Janser, R. F. J.; Fairbanks, S. S.; Vortolomei, C. M.; Meka, R. K.; Janser, I. Selective methoxy ether cleavage of 2,6dimethoxyphenol followed by a selective acylation. Tetrahedron Lett. 2012, 53, 11-14.

(18) McOmie, J. F. W.; Watts, M. L.; West, D. E. Demethylation of aryl methyl ethers by boron tribromide. Tetrahedron 1968, 24, 22892292.

(19) Tachrim, Z.; Wang, L.; Murai, Y.; Yoshida, T.; Kurokawa, N.; Ohashi, F.; Hashidoko, Y.; Hashimoto, M. Trifluoromethanesulfonic Acid as Acylation Catalyst: Special Feature for C- and/or O-Acylation Reactions. Catalysts 2017, 7, 40.

(20) Plażuk, D.; Zakrzewski, J. Friedel-Crafts acylation of ferrocene with alkynoic acids. J. Organomet. Chem. 2009, 694, 1802-1806.

(21) Padhye, S.; Ahmad, A.; Oswal, N.; Sarkar, F. H. J. Hematol. Oncol. 2009, 2 (38), 38.

(22) Rabinovich, D. Topocehmistry. Part XXX. Crystal and molecular structures of chalcone. J. Chem. Soc. B 1970, 0, 11-16.

(23) Rao, S. S.; O’Neil, J.; Liberator, C. D.; Hardwick, J. S.; Dai, X.; Zhang, T.; Tyminski, E.; Yuan, J.; Kohl, N. E.; Richon, V. M.; Van der Ploeg, L. H.; Carroll, P. M.; Draetta, G. F.; Look, A. T.; Strack, P. R.; Winter, C. G. Inhibition of NOTCH signaling by gamma secretase inhibitor engages the RB pathway and elicits cell cycle exit in T-cell acute lymphoblastic leukemia cells. Cancer Res. 2009, 69 (7), 3060-8.

(24) Niimi, H.; Pardali, K.; Vanlandewijck, M.; Heldin, C. H.; Moustakas, A. Notch signaling is necessary for epithelial growth arrest by TGF-beta. J. Cell Biol. 2007, 176, 695-707. 